\title{
TET1 may contribute to hypoxia-induced epithelial to mesenchymal transition of endometrial epithelial cells in endometriosis
}

\author{
Jingni Wu ${ }^{1}$, Xidie Li ${ }^{1}$, Hongyan Huang ${ }^{1}$, Xiaomeng Xia ${ }^{1}$, Mengmeng Zhang ${ }^{1}$, Xiaoling Fang ${ }^{\text {Corresp. } 1}$ \\ ${ }^{1}$ Department of Obstetrics and Gynecology, The Second Xiangya Hospital, Central South University, Changsha, Hunan, China \\ Corresponding Author: Xiaoling Fang \\ Email address: fxlfx10510@csu.edu.cn
}

Background. Endometriosis (EMs) is a non-malignant gynecological disease, whose pathogenesis remains to be clarified. Recent studies have found that hypoxia induces epithelial-mesenchymal transition (EMT) as well as epigenetic modification in EMs. However, the relationship between EMT and demethylation modification under hypoxia status in EMs remains unknown.

Methods. The expression of N-cadherin, E-cadherin and TET1 in normal endometria, eutopic endometria and ovarian endometriomas was assessed by immunohistochemistry and immunofluorescence double staining. 5-hmC was detected by fluorescence-based ELISA kit using a specific 5-hmC antibody.

Overexpression and inhibition of TET1 or hypoxia-inducible factor $2 \alpha$ (HIF-2 $\alpha$ ) were performed by plasmid and siRNA transfection. The expression of HIF-2 $\alpha$, TET1 and EMT markers in Ishikawa (ISK) cells (widely used as endometrial epithelial cells) was evaluated by western blotting. The interaction of HIF-2 $\alpha$ and TET1 was analyzed by chromatin immunoprecipitation.

Results. Demethylation enzyme TET1 (ten-eleven translocation1) was elevated in glandular epithelium of ovarian endometrioma, along with the activation of EMT (increased expression of N-cadherin, and decreased expression of E-cadherin) and global increase of epigenetic modification marker 5-hmC(5hydroxymethylcytosine). Besides, endometriosis lesions had more TET1 and N-cadherin co-localized cells. Further study showed that ISK cells exhibited enhanced EMT, and increased expression of TET1 and HIF-2 $\alpha$ under hypoxic condition. Hypoxia-induced EMT was partly regulated by TET1 and HIF-2 $\alpha$. HIF- $2 \alpha$ inhibition mitigated TET1 expression changes provoked by hypoxia.

Conclusions. Hypoxia induces the expression of TET1 regulated by HIF- $2 \alpha$, thus may promote EMT in endometriosis. 
1 TET1 may contribute to hypoxia-induced epithelial to mesenchymal transition of

2 endometrial epithelial cells in endometriosis

3 Jingni $\mathrm{Wu}^{1}$, Xidie $\mathrm{Li}^{1}$, Hongyan Huang ${ }^{1}$, Xiaomeng Xia ${ }^{1}$, Mengmeng Zhang ${ }^{1}$, Xiaoling Fang ${ }^{1 *}$

51 Department of Obstetrics and Gynecology, The Second Xiangya Hospital, Central South

6 University, Changsha 410011, China

7 Corresponding Author: Email: fxlfx10510@csu.edu.cn

\section{Abstract}

Background. Endometriosis (EMs) is a non-malignant gynecological disease, whose pathogenesis

11 remains to be clarified. Recent studies have found that hypoxia induces epithelial-mesenchymal

12 transition (EMT) as well as epigenetic modification in EMs. However, the relationship between

13 EMT and demethylation modification under hypoxia status in EMs remains unknown.

14 Methods. The expression of N-cadherin, E-cadherin and TET1 in normal endometria, eutopic

15 endometria and ovarian endometriomas was assessed by immunohistochemistry and

16 immunofluorescence double staining. 5-hmC was detected by fluorescence-based ELISA kit using

17 a specific 5-hmC antibody. Overexpression and inhibition of TET1 or hypoxia - inducible factor

$182 \alpha($ HIF- $2 \alpha)$ were performed by plasmid and siRNA transfection. The expression of HIF-2 $\alpha$, TET1

19 and EMT markers in Ishikawa (ISK) cells (widely used as endometrial epithelial cells) was

20 evaluated by western blotting. The interaction of HIF-2 $\alpha$ and TET1 was analyzed by chromatin

21 immunoprecipitation. 
22 Results. Demethylation enzyme TET1 (ten-eleven translocation1) was elevated in glandular epithelium of ovarian endometrioma, along with the activation of EMT (increased expression of $\mathrm{N}$-cadherin, and decreased expression of E-cadherin) and global increase of epigenetic modification marker 5-hmC(5-hydroxymethylcytosine). Besides, endometriosis lesions had more TET1 and N-cadherin co-localized cells. Further study showed that ISK cells exhibited enhanced EMT, and increased expression of TET1 and HIF-2 $\alpha$ under hypoxic condition. Hypoxia-induced EMT was partly regulated by TET1 and HIF-2 $\alpha$. HIF-2 $\alpha$ inhibition mitigated TET1 expression changes provoked by hypoxia.

Conclusions. Hypoxia induces the expression of TET1 regulated by HIF-2 $\alpha$, thus may promote EMT in endometriosis.

\section{Keywords}

Endometriosis, TET1, EMT, HIF-2 $\alpha$

\section{Introduction}

Endometriosis is a chronic and non-malignant gynecological disease characterized by the growth of endometrial glands and stroma outside the uterus (Giudice, 2010). It exhibits cancer-like features, such as cell proliferation and metastatic invasion. Endometriosis is a major contributor to pelvic pain and infertility (Mahmood \& Templeton, 1991). Although the etiology of endometriosis

41 is still unclear, retrograde menstrual reflux is the widely accepted hypothesis for the mechanism of endometriosis. This theory states that retrograded endometrial tissues must migrate, invade and 
survive outside the cavity of uterus, then establish new endometriosis lesions. However, little is known about the molecular events that lead to the development of endometriosis.

Due to lack of hormone and blood supply, the retrograded endometrial debris during menstruation is in hypoxic status (Maybin \& Critchley, 2015). Hypoxia plays a role in the migration and invasive of endometrial epithelial cells in endometriosis (Xiong et al., 2016). It probably induces the survival of retrograded endometrial debris and angiogenesis in implanted ectopic endometrial lesions ( $\mathrm{Wu}$ et al., 2007). That is, the hypoxia microenvironment may contribute to the migration, invasion, and ectopic implant formation of the eutopic endometrial epithelial cells. Therefore, hypoxia has been regarded as an important stimulus of the pathological process of endometriosis. Hypoxia can stabilize hypoxia-inducible factors (HIFs, including HIF$1 \alpha$ and HIF-2 $\alpha$ ), which are the most significant and sensitive mediators of hypoxia-induced cellular responses. Stabilized HIFs dimerize with their constitutively stabilized partner, HIF-1 $1 \beta$, and regulate the expression of the target gene, thereby leading to hypoxia-induced phenotypes (Hsiao, 2015; Jain et al., 2018). However, few studies have investigated the role of the transcription factor HIF-2 $\alpha$ in endometriosis.

Studies have shown that hypoxia promotes the epithelial to mesenchymal transition (EMT) and enhances endometrial cell migration and invasion in endometriosis (Liu et al., 2017, 2018). EMT is a process by which epithelial cells lose polarity and cell-to-cell contacts and transform into mesenchymal cells with high motility. This process is characterized by the downregulation of the epithelial marker, E-cadherin, and the upregulation of mesenchymal markers, N-cadherin and vimentin (Lamouille, Xu \& Derynck, 2014), which may occur in EMs (Matsuzaki \& Darcha, 
64 2012). EMT endows cells with migratory and invasive properties, a prerequisite for the

65 establishment of endometriotic lesions (Matsuzaki \& Darcha, 2012; Xiong et al., 2016).

66 Epigenetic alterations of chromatin (including DNA methylation, histone modifications, and

67 non-coding RNAs regulation) are proposed to facilitate EMT in many diseases. Aberrations in

68 DNA methylation are associated with EMT and tumorigenesis under hypoxia conditions (Camuzi

69 et al., 2019). However, in endometriosis, the relationship between EMT and DNA methylation

70 under hypoxia status remains largely unknown. The dynamic balance between methylation and

71 demethylation is crucial for various biological processes (Jones, 2012). The altered expression of

72 the demethylation enzyme ten-eleven translocation (TET1) disrupts this balance (Lorsbach et al.,

73 2003), leading to aberrant DNA methylation patterns, which is seen in many human diseases, such

74 as cancer (Baylin \& Jones, 2011). TET1 enzymes iteratively oxidize 5-methylcytosine (5-mC) to

75 5-hydroxymethylcytosine (5-hmC), thereby contributing to $\mathrm{CpG}$ island demethylation in specific

76 gene promoters (Wu \& Zhang, 2017). TET1 is involved in cell migration, differentiation, and

77 oncogenesis (Yang et al., 2015). A recent study has shown that TET genes are dysregulated in

78 endometriosis (Roca et al., 2016). However, the function of TET1 in endometriosis is not yet fully

79 understood.

80 Our study investigated for the first time the role and mechanism of TET1 in regulating the

81 EMT process of endometrial epithelial cells under hypoxic conditions. We hypothesized that

82 hypoxia may increase TET1 expression, mediated by HIF-2 $\alpha$, thus potentially inducing the EMT

83 of endometrial epithelial cells and contributing to the development of endometriosis. An

84 infographic of this work is shown in Figure 1.

Peer] reviewing PDF | (2020:04:47385:1:2:NEW 12 Aug 2020) 
Materials and methods

86

87

\section{Patients and sample collection}

The patients recruited in the study were women of childbearing age from the Second Xiangya Hospital. Patients with any hormonal-dependent disease, irregular menstrual cycles and those who took steroids and GnRH agonists for the past six months were excluded. The endometrial samples were obtained from these patients and were all confirmed in the early proliferation stage by pathological examination. This study was approved by the Human Ethics Committee of Second Xiangya Hospital, Central South University. Written informed consent was obtained from all the patients (Ref. No. 2016-243).

Different types of endometriotic lesions may have different pathogeneses (Nisolle \& Donnez, 1997). Ovarian endometriosis lesions are more correlated with hypoxia and angiogenic factors (Filippi et al., 2016). Hence, we focused on the ovarian endometrioma in this study. Fifteen ovarian endometriomas and fifteen eutopic endometria (from the same group of women with ovarian endometriosis), and fifteen samples of normal endometria were used for the immunohistochemistry. The stages of endometriosis were classified according to the rASRM classification criteria during the procedure of laparoscopy. Clinical characteristics of the patients are shown in Table 1. Besides, these samples were fixed in $10 \%$ buffered formalin and embedded in paraffin for immunohistochemistry analysis.

\section{Immunohistochemistry (IHC)}

Immunohistochemistry staining was performed on paraffin-embedded blocks of eutopic endometria, ovarian endometriomas and normal endometria.5- $\mu \mathrm{m}$-thick tissue sections were 
106

107

108

109

110

111

112

113

114

115

116

117

118

119

120

121

122

123

124

125

126

deparaffinized in xylene and rehydrated in graded ethanol, and antigen retrieval was performed in $0.01 \mathrm{~mol} / 1$ sodium citrate buffer $(\mathrm{pH} 6.0)$ using a pressure cooker. Sections were then treated with 3\% hydrogen peroxide for 10 min to block endogenous peroxidase. After rinsing in PBST, sections

were blocked in $5 \%$ bovine serum albumin for $30 \mathrm{~min}$, then incubated overnight at $4{ }^{\circ} \mathrm{C}$ with primary antibodies. The second day, sections were incubated with horseradish peroxidase (HRP)conjugated secondary anti-rabbit IgG for $30 \mathrm{~min}$ after rinsing for three times. Antigen-antibody reactions were detected by the HRP-catalyzed reaction with 3,3'-diaminobenzidine (DAB) (Beyotime Biotechnology, Shanghai, China). Finally, the sections were counterstained with hematoxylin and analyzed by optical microscopy. Tyramide signal amplification (TSA) technique was used to enhance the sensitivity of IHC. The following primary antibodies were applied: Ncadherin (\#66219-1-AP, proteintech, Wuhan, China, 1:1000), E-cadherin (\#20874-1-AP, proteintech, Wuhan, China, 1:2000) and TET1 (ab191698, Abcam, Cambridge, UK, 1:1000).

\section{DNA isolation and determination of global 5-hmC levels}

Genomic DNA from human endometrium and endometriosis lesions were isolated and purified using the CWBIO Genomic DNA kit according to the manufacturer's instructions. The concentration and quality of the DNA were estimated by agarose electrophoresis and UV absorption analysis. Global 5-hmC levels of this isolated genomic DNA were determined with a fluorescence-based Enzyme Linked Immunosorbent Assay (ELISA) kit using a specific 5-hmC antibody (Epigentek, Farmingdale, NY, USA), according to the manufacturer's recommendations. $200 \mathrm{ng}$ of total genomic DNA was applied for this assay. The 5-hmC quantities in these DNA samples were analyzed based on the OD value generated by a microplate reader compared to 
127

128

129

130

131

132

133

134

135

136

137

control DNA in the kit. All samples were run in duplicate. These data were obtained from six normal endometria and six eutopic endometria and six ovarian endometriomas.

\section{Immunofluorescence double staining}

Paraffin-embedded sections were deparaffinised, rehydrated, heated in $0.01 \mathrm{~mol} / \mathrm{l}$ sodium citrate buffer ( $\mathrm{pH}$ 6.0) for antigen retrieval, and treated with 3\% hydrogen peroxide for 10 min to block endogenous peroxidase activity. After washing with PBS three times and blocking in 5\% bovine serum albumin for $30 \mathrm{~min}$, the sections were incubated in the primary antibody solution containing the rabbit anti-TET1 antibody (1:1000), rabbit anti-N-Cadherin antibody (1:200), and rabbit antiE-cadherin antibody (1:50). Because we used TSA technique to enhance the fluorescent signal, antibodies of the same species were acceptable in our experiment. The biotinylated secondary antirabbit antibody was used to detect primary antibodies. The detection was performed with Streptavidin-HRP D (DAB Map kit, Ventana Medical Systems), followed by incubation with 488 (cat\# T20922) or 594 (cat\# T20935) Tyramide Alexa Fluors (Invitrogen) prepared in the dark according to the manufacturer's instructions. Sections were counterstained with DAPI.

\section{Image analysis}

Immunohistochemistry and immunofluorescence double staining experiments were analyzed with a PerkinElmer Quantitative Pathology Imaging System in the Hunan Epigenetics Laboratory. This imaging system includes the Mantra Quantitative Pathology Workstation (PerkinElmer, CLS140089) for collecting staining data and PerkinElmer inForm software (PerkinElmer, Hopkinton, MA, USA) for analysis. The scanned images were visually examined. The samples with staining artifacts or with low quality were excluded. Image processing comprised the training 
session and analysis session. InForm software was trained to identify regions of interest and used to analyze the whole image. Three fields of view were randomly selected per slide to calculate the expression levels and distribution of TET1, E-cadherin, and N-cadherin in the glandular epithelium. The proportion of positive cells is calculated as the number of positive-staining cells divided by the number of total glandular epithelial cells. The correlation between TET1 and EMT markers expression in the endometrial epithelium (positive cell proportion) was analyzed by

Pearson correlation test.

\section{Cell culture and hypoxia treatment}

Ishikawa (ISK) cells (ZQ0472, Zhong Qiao Xin Zhou Biotechnology Co., Ltd, Shanghai, China) come from well-differentiated human endometrial adenocarcinoma. The human endometrial epithelial cell is hard to passage and transfect; endometrial stromal cells are the only cells to survive after 3-4 generations in vitro. Therefore, ISK cells are widely used instead of human endometrial glandular epithelial cells in studies of endometriosis (Guay \& Akoum, 2007; Cho et al., 2016). ISK cells were cultured in Dulbecco's Modified Eagle's Medium/Nutrient Mixture F12 (DMEM/F12) supplemented with 10\% fetal bovine serum (FBS) and 1\% penicillinstreptomycin in $5 \% \mathrm{CO}_{2}$ incubators at $37^{\circ} \mathrm{C}$ until $70 \%$ confluence was reached. To ensure adequate nutrients and growth factors, the medium was renewed $1 \mathrm{~h}$ prior to hypoxia treatment. Thereafter, ISK cells were cultured under hypoxia $\left(5 \% \mathrm{O}_{2}\right)$ or normoxia $\left(20 \% \mathrm{O}_{2}\right)$ conditions for 4 , 8, and 24 h.

\section{Gene transfection}

ISK cells were seeded in six-well plates for $24 \mathrm{~h}$ to reach approximately $70 \%$ confluence and 
169

170

171

172

173

174

175

176

transfected with TET1 or the negative control plasmid (GenScript Biotech Corporation; Piscataway, NJ, USA) using Lipofectamine (Invitrogen, Carlsbad, CA, USA), according to the manufacturer's protocol. In Brief, we used the QIAGEN ${ }^{\mathrm{R}}$ kit to purify and extract the plasmid. Then, 1-4 $\mu \mathrm{g}$ of plasmid DNA and Lipofectamine solution were mixed in serum-reduced medium and added to the ISK cells. After $6 \mathrm{~h}$ of incubation, fresh medium was added. For TET1 downregulation, TET1-targeting siRNAs and the negative control (NC) were obtained from Ribobio (Guangzhou, China). The RNA interference (RNAi) experiments were performed following the manufacturer's protocol.First, the TET1 siRNA and NC siRNAs were each mixed with RNA transfection buffer (Ribo FECT CP) and then added to ISK cell medium. After $48 \mathrm{~h}$, western blotting was performed to determine the transfection efficiency.

\section{Protein extraction and western blotting analysis}

Total proteins were extracted from cells using RIPA lysis buffer and phosphatase inhibitors. All lysates were centrifuged at $12,000 \times \mathrm{g}$ for $20 \mathrm{~min}$. The supernatants were collected, and the protein concentrations were determined by the Bicinchonic Acid (BCA) protein assay kit (Beyotime Biotechnology, Shanghai, China). Proteins were mixed with the same amount of loading buffer and boiled for $5 \mathrm{~min}$. The samples were then resolved by Sodium dodecyl-sulfate polyacrylamide gel electrophoresis (SDS-PAGE), transferred to PVDF membranes, incubated with 5\% fat-free milk, and hatched with the following primary antibodies at $4^{\circ} \mathrm{C}$ overnight: $\mathrm{N}$-cadherin, E-cadherin, TET1, HIF-2 $\alpha$, and HIF-1 $\alpha$ (Abcam, Cambridge, UK). The membranes were washed with TBST three times for $15 \mathrm{~min}$, and then incubated in a secondary antibody at $37^{\circ} \mathrm{C}$ for $1.5 \mathrm{~h}$. The blots were analyzed by a chemiluminescence system (Millipore. USA). These experiments were 
190

191

192

193

194

195

196

197

198

199

200

201

202

203

204

205

206

207

208

209

210

repeated three times, and the average values of the blot bands were calculated.

\section{Co-immunoprecipitation}

Co-immunoprecipitation was carried out to identify protein-protein interactions. Cells were collected after exposure to hypoxia. Cell pellets were lysed in RIPA lysis buffer containing phosphatase inhibitors. $750 \mu \mathrm{l}$ of the cell lysates were incubated with $2 \mu \mathrm{g}$ of antibodies (rabbit HIF-2 $\alpha$, mouse HIF-1 $\alpha$, and rabbit TET1) overnight at $4^{\circ} \mathrm{C}$. Meanwhile, $\beta$-actin, rabbit IgG, and mouse $\lg \mathrm{G}$ (Proteintech, Wuhan, China) were used as the input and negative controls, respectively, for the experiments. On the second day, each group was mixed with $20 \mu$ l of Protein G Plus/Protein A Agarose Suspension (Santa Cruz Biotechnology, Santa Cruz, CA, USA) and and the solution was softly shook at $4^{\circ} \mathrm{C}$ for $2 \mathrm{~h}$. To separate agarose beads, these mixtures were centrifuged at $3000 \mathrm{rpm}$ for $3 \mathrm{~min}$ at $4^{\circ} \mathrm{C}$. The supernatant was transferred to a new tube on ice, and the beads were eluted again with lysis buffer. The total eluted supernatants were washed 5 times, resuspended in $1 \times$ loading buffer, and boiled for $5 \mathrm{~min}$ at $100^{\circ} \mathrm{C}$ before western blotting.

\section{Statistical analysis}

Statistical analysis of data was performed by one-way ANOVA using SPSS 22.0 software (SPSS Inc., Chicago, IL, USA). All experiments were repeated three times. The results are shown as the mean \pm standard deviation (SD). A P value of $<0.05$ was considered statistically significant.

\section{Results}

Accompanied with TET1 upregulation, EMT might occur in endometrial epithelial cells of ovarian endometriosis 
211 To evaluate the expression and localization of TET1 and EMT markers, E-cadherin and N-

212 cadherin, in endometriosis, we performed IHC and immunofluorescence double staining in human

213 endometrial tissues. Since the morphology of stromal cells in the ovarian endometriomas is much

214 different from that in the normal endometria and eutopic endometria, we only analyzed the 215 glandular epithelial cells in the three groups.

216 The expression of TET1 and EMT markers in the glandular epithelium of normal endometria, eutopic endometria, and ovarian endometriomas was analyzed by IHC. Figure 2A-R show the representative IHC results. Table 2 illustrates the expression of TET1 and EMT markers. The proportions of cells positively stained for TET1 or N-cadherin in the glandular epithelium of the eutopic endometria and ovarian endometriomas were significantly higher than those in the normal endometria, and the number of E-cadherin-positive cells was significantly lower in the glandular epithelium from eutopic endometria and ovarian endometriomas than those in the normal endometria (Figure 2S-U). The normal ovary tissue has a low expression of TET1 (supplementary Figure 1). Moreover, to demonstrate whether the demethylation enzyme TET1 was activated in endometriosis, we explored the expression of $5-\mathrm{hmC}$, which is an important marker for the activated demethylation process. Figure 2V shows that the ovarian endometriomas have higher 5hmC levels $(5.15 \pm 1.488 \%$ of total DNA) than eutopic endometria $(3.33 \pm 0.876 \%$ of total DNA, $\mathrm{p}<0.05)$ and normal endometria $(3.5 \pm 0.881 \%$ of total DNA, $\mathrm{p}<0.05)$. All in all, these data suggest that TET1 is upregulated and EMT may occur in the glandular epithelium of endometriosis.

Immunofluorescence double staining was performed to compare the localization of TET1 and EMT markers. Consistent with the IHC results, in eutopic endometria and normal endometria, 
232 TET1 and N-cadherin were mainly expressed in the stromal cells and barely expressed in the 233 glandular epithelial cells. However, in the ovarian endometriomas, TET1 and N-cadherin were 234 both expressed in the glandular epithelial cells. Furthermore, the distribution of TET1 was more

obvious in the cytoplasm or nuclear of N-cadherin-positive cells (Figure 2FF-NN), not Ecadherin-positive cells (Figure 2W-EE). Ovarian endometriotic lesions (Figure 2LL-NN) had more TET1 and N-cadherin co-localized cells compared to eutopic endometria (Figure 2II-KK) and normal endometria (Figure 2FF-HH). The large amount of co-localized cells suggests a close relationship between TET1 and N-cadherin. In addition, a correlation analysis was applied to the proportions of cells that were positively stained for TET1 and N-cadherin/E-cadherin in the epithelium (Figure 2OO-PP), which showed that TET1 was positively correlated with N-cadherin and negatively correlated with E-cadherin. Our findings suggest that the demethylation enzyme TET1 may play a role in the EMT pathological process of endometriosis.

\section{Hypoxia alters the expression of HIFs, TET1 and EMT markers}

Although various epigenetic mechanisms have been demonstrated to regulate EMT under hypoxia status (Wu et al., 2012; Tsai \& Wu, 2014), the role of the DNA demethylation enzyme TET1 in regulating hypoxia-induced EMT in endometriosis remains largely unknown. Therefore, we investigated the expression of TET1, HIFs (HIF-1 $\alpha$ and HIF-2 $\alpha$ ), and EMT markers (E-cadherin, $\mathrm{N}$-cadherin, and vimentin) in ISK cells exposed to $5 \% \mathrm{O}_{2}$ for $0 \mathrm{~h}, 4 \mathrm{~h}, 8 \mathrm{~h}$ and $24 \mathrm{~h}$. As shown in Figure 3, the expression of TET1, HIF-1 $\alpha$, HIF-2 $\alpha$, and vimentin increased significantly and the expression of E-cadherin decreased significantly after hypoxia treatment for $8 \mathrm{~h}$ and $24 \mathrm{~h}$, especially for $24 \mathrm{~h}$. $\mathrm{N}$-cadherin expression peaked at $8 \mathrm{~h}$ after hypoxic treatment and went down 
253 at $24 \mathrm{~h}$, we presumed $\mathrm{N}$-cadherin might be transiently triggered by hypoxia. Homeostasis should 254 work to reverse the hypoxia condition, and genes may be activated in different time period of 255 hypoxia during this process (Deret et al., 2004; Javaid et al., 2013; Song et al., 2019). Hence, the

256

257

258

259

260

261

262

263

264

265

266

267

268

269

270

271

272

273

time point with the maximal response was selected, and vimentin was chosen as a mesenchymal

marker in the following experiences. Our findings indicate that hypoxia activates TET1 expression and the EMT.

TET1 overexpression may promote EMT, and knockdown of TET1 mitigates hypoxiainduced EMT in ISK cells

To evaluate the role of TET1 in hypoxia-induced endometrial EMT, we successfully established

TET1-overexpressing ISK cells under normal conditions and TET1 down-regulation ISK cells under hypoxia conditions. As seen in Figure 4, western blotting indicated that the expression of vimentin was increased and the expression of E-cadherin was decreased by upregulation of TET1 under normoxia. In addition, hypoxia-induced EMT, characterized by the repression of E-cadherin and the upregulation of vimentin, was abolished by TET1 knockdown. These results reveal that TET1 plays a crucial role in hypoxia-induced EMT.

\section{Knockdown of HIF-2 $\alpha$ inhibits hypoxia-induced EMT and TET1 expression in ISK cells}

To investigate the mechanism of TET1 in the regulation of hypoxia-induced EMT, hypoxiainduced HIF-2 $\alpha$ expression was inhibited by a specific siRNA for $48 \mathrm{~h}$. Western blotting analysis confirmed the down-expression of HIF-2 $\alpha$. Knockdown of HIF-2 $\alpha$ mitigated the activation of TET1 and EMT under hypoxia in ISK cells (Figure 5A and Figure 5C-F), indicating that HIF$2 \alpha$ is a regulator of TET1 expression and the EMT under hypoxia. Xiong et al. reported that HIF- 
$2741 \alpha$ also induced EMT in endometrial epithelial cells under hypoxia (Xiong et al., 2016). Therefore,

275 we conducted chromatin immunoprecipitation experiments, which showed that the anti-TET1

276 antibody pulled down HIF-2 $\alpha$ and TET1, not HIF-1 $\alpha$, and the anti-HIF-2 $\alpha$ antibody pulled down

277 both TET1 and HIF-2 $\alpha$. This implied that HIF-2 $\alpha$, not HIF-1 $\alpha$, is directly or indirectly bound to

278 the TET1 protein (Figure 5B). Reporter gene assay demonstrated that the promoter region of the

279 TET1 gene was activated by hypoxia/HIF-2 $\alpha$ (Tsai et al., 2014). All these results indicate that HIF-

$2 \alpha$ may serve as a transcription activator in ISK cells to regulate TET1 involving hypoxia-induced

EMT.

\section{Discussion}

Aberrant DNA methylation may be associated with the pathogenesis of endometriosis (Arosh et

al., 2015; Koukoura, Sifakis \& Spandidos, 2016; Li et al., 2017; Juanqing et al., 2019). TET1-

mediated DNA hydroxymethylation is a crucial mechanism of DNA demethylation (Jeschke,

Collignon \& Fuks, 2016). The balance between methylation and demethylation is important for

gene expression and cellular environmental homeostasis (Song \& He, 2012; Yang et al., 2015).

However, the demethylation modification in endometriosis has not yet been well studied. Our

study shows for the first time that the elevated expression of the demethylation enzyme TET1 may

be associated with the activated EMT phenotype in the epithelia of endometriotic lesions, which may provide a novel insight into the pathogenesis of EMs.

293 Genome-wide DNA hypomethylation occurs in many diseases (Ehrlich, 2009; Song \& He, 
295 role in regulating gene expression (Lorsbach et al., 2003; Mariani et al., 2014; Jeschke, Collignon

296 \& Fuks, 2016). Our study had some consistency with the report by Roca (Roca et al., 2016).

297 Although Roca et al. showed that TET1 was down-regulated in endometriotic tissues, the contribution of TET1 expression from each component of the endometrium (epithelium or stroma) is unknown. Endometriosis is an estrogen-dependent disease. Roca et al. found that global 5-hmC was upregulated in endometriotic tissues and TET1 was upregulated in endometrial epithelial cell line treated with estradiol, which supports our study and suggests that TET1 in epithelial cells may affect the expression levels of 5-hmC globally or locally. It is worth noting that the stromal cells are more loosely arranged in the ovarian endometriomas compared to the normal endometria and eutopic endometria. In addition, the endometriotic stromal and epithelial cells have different molecular expression profiles (Logan, Yango \& Tran, 2018; Noë et al., 2018), and the endometriotic epithelial cells might originate from endometrial epithelial cells, whereas the origin of endometriotic stromal cells remains to be investigated (Matsuzaki \& Darcha, 2012). Hence, we focused on the endometrial epithelium, which might play a specific and dynamic role in the pathogenesis of endometriosis (Logan, Yango \& Tran, 2018; Noë et al., 2018). A recent study showed that decreased TET1 expression led to 5-hmC loss in ISK cells (Lv et al., 2017). We need to explore the distribution of $5 \mathrm{hmC}$ in endometriosis, especially in the epithelia, and the specific gene whose promoter is enriched with $5-\mathrm{hmC}$ in future studies.

TET1 was mainly expressed in stromal cells but not epithelial cells in the normal and eutopic endometria, whereas the epithelial expression of TET1 expression was ubiquitous and stromal 
inverse expression between TET1 and the epithelial marker E-cadherin and a positive correlation

317 between the expression of TET1 and the mesenchymal marker N-cadherin. Therefore, our study

318 demonstrates for the first time that the enhanced epithelial expression of TET1 in ectopic lesions

319 may play a crucial role in the EMT phenotype of endometriosis while the role of TET1 in stromal cells needs to be further investigated. Our research may lay the foundation for this new demethylation mechanism of endometriosis. The localization of TET1 in both the nucleus and cytoplasm was surprising since TET1 is a nuclear protein. However, the similar subcellular localization of TET1 was also shown in prostate, gastric cancer, hippocampus neurons and so on

(Hsu et al., 2012; Kaas et al., 2013; Fu et al., 2014; Han et al., 2017). TET genes localize in the

cytoplasm of neurons to sustain cell survival (Mi et al., 2015) and localize in the cytoplasm of colorectal cancer cells to promote tumor metastasis (Huang et al., 2016). The variations of TET1 immunostaining results indicate a possible regulation of TET1 subcellular localization by still unclear signaling pathways.

Hypoxia is an important factor of the endometriosis microenvironment (Becker et al., 2008;

Wu, Hsiao \& Tsai, 2019). Hypoxia can induce epigenetic changes in tumor cells, whereas hypoxiainduced epigenetic changes in endometriosis haven't been reported yet (Zhou et al., 2006;

Shahrzad et al., 2007). In our study, we showed for the first time that hypoxia/HIF-2 $\alpha$ activated

TET1 expression, and TET1 knockdown mitigated hypoxia-induced EMT in ISK cells. Coimmunoprecipitation revealed that HIF-2 $\alpha$, not HIF-1 $\alpha$, was bound to the TET1 protein in ISK cells. Therefore, we did not further explore the role of HIF-1 $\alpha$ in our study. This co- 
337 (Tsai et al., 2014) and Cheng et al. (Cheng et al., 2018). Tsai et al. showed the interaction of TET1

338 and HIF-1a based on the $293 \mathrm{~T}$ cell line while Cheng et al. showed the same interaction based on

339 the cells in mouse prefrontal cortex. Whereas, our study was performed on the ISK cells which

340 could have different protein-protein interactions. What's more, Tsai et al. concluded that HIF-2 $\alpha$

341 was the major regulator of TET1 expression under hypoxia (Tsai et al., 2014). In this study, the

342 HIF-2a knockdown didn't eliminate the expression of TET1, which might indicate the existence

343 of other regulators. Hu et al. reported microRNA-210 was involved in the regulation of TET1

344 under hypoxia status (Hu et al., 2018). Lin et al. showed HIF-1a could regulate the hypoxia-

345 induced expression of TET in hepatoblastoma HepG2 cells (Lin et al., 2017), whereas Tsai et al.

346 showed the HIF-1a knockdown had no effect on the TET1 expression in cancer cell lines (Tsai et al., 2014). It will be explored in future studies. Combined with the reporter gene assay of HIF-2 $\alpha$ and TET1 (Tsai et al., 2014), we speculate that the hypoxia microenvironment may induce the EMT of endometrial epithelial cells via the activation of TET1 partly regulated by HIF-2 $\alpha$. These epithelial cells may pass through oviducts and implant into the ovarian surfaces, thus contributing to the development of endometriosis.

Our study had several limitations. First, we used ISK cells for in vitro experiments instead of the primary endometrial epithelial cells. The primary endometrial epithelial cells could hardly survive in primary culture. Therefore, ISK cells were used in numerous studies of endometriosis

(Cho et al., 2016b; Lee et al., 2018; Matson et al., 2018; Choi et al., 2018). The ISK cell is a welldifferentiated human endometrial adenocarcinoma cell line. It retains the phenotype of endometrial epithelial cells, bears estrogen and progesterone receptors, and displays a similar molecular 
358

360

361

362

363

364

365

366

367

368

369

370

371

372

373

374

375

376

377

378

expression profile as endometrium (Du et al., 2018). However, our study will be more convincing if we use more cell lines. With the advancement of technology and improvement of experimental approaches, a deep understanding of the roles of TET1 in endometriosis will be possible. Secondly, the precise role and mechanism of TET1 in regulating the EMT remain to be investigated. The TET1 target gene and the correlation of TET1 with the EMT transcriptional factors slug, snails, and twist need to be elucidated in future studies. The interaction between TET 1 and HIF-2 $\alpha$ needs to be demonstrated by additional experiments, such as GST pull-down and yeast two-hybrid experiments. Thirdly, although $5-\mathrm{hmC}$ detection is very expensive, analyzing more samples for overall 5-hmC expression in the epithelia and identifying the specific gene enriched with 5-hmC are still needed to study the pathogenesis of endometriosis.

\section{Conclusion}

To our knowledge, we are the first to explore the relationship between EMT and the demethylation enzyme TET1 in endometriosis. Hypoxia induces the expression of TET1, mediated by transcription factor HIF-2 $\alpha$, which may promote the EMT of endometriosis. These data provide a new understanding of the pathological process of endometriosis, which may advance knowledge of the epigenetic mechanism as well as the therapeutic approach towards endometriosis.

\section{Authors' Contribution}

J.W., X.L., X.F. conceived and designed the experiments. J.W., X.L., H.H., M.Z., H.H., X.F. 
performed and acquired the data. J.W., X.X., X.F. analyzed the data. J.W., X.L., H.H., X.F. drafted and critically evaluated the article.

\section{Funding}

This work was supported by the National Natural Science Foundation of China [grant number

China [grant number 2020zzts285].

\section{Conflict of interest}

The authors declare no conflict of interest regarding this paper.

\section{Acknowledgments}

None

\section{References}

Arosh JA, Lee J, Starzinski-Powitz A, Banu SK. 2015. Selective inhibition of prostaglandin E2 receptors EP2 and EP4 modulates DNA methylation and histone modification machinery proteins in human endometriotic cells. Molecular and cellular endocrinology 409:51-58.

Baylin SB, Jones PA. 2011. A decade of exploring the cancer epigenome - biological and translational implications. Nature Reviews. Cancer 11:726-734. DOI: 10.1038/nrc3130. 
400 Becker CM, Rohwer N, Funakoshi T, Cramer T, Bernhardt W, Birsner A, Folkman J, D’Amato RJ. 2008. 2-

401

402

403

404

405

406

407

408

409

410

411

412

413

414

415

416

417

418

419

420
Methoxyestradiol Inhibits Hypoxia-Inducible Factor-1 $\alpha$ and Suppresses Growth of Lesions in a Mouse

Model of Endometriosis. The American Journal of Pathology 172:534-544. DOI:

10.2353/ajpath.2008.061244.

Camuzi D, de Amorim ÍSS, Ribeiro Pinto LF, Oliveira Trivilin L, Mencalha AL, Soares Lima SC. 2019. Regulation Is in the Air: The Relationship between Hypoxia and Epigenetics in Cancer. Cells 8:300.

Cheng Y, Sun M, Chen L, Li Y, Lin L, Yao B, Li Z, Wang Z, Chen J, Miao Z, Xin N, Huang L, Allen EG, Wu H, Xu X, Jin P. 2018. Ten-Eleven Translocation Proteins Modulate the Response to Environmental Stress in Mice. Cell Reports 25:3194-3203.e4. DOI: 10.1016/j.celrep.2018.11.061.

Cho S, Mutlu L, Zhou Y, Taylor HS. 2016a. Aromatase inhibitor regulates let-7 expression and let-7f-induced cell migration in endometrial cells from women with endometriosis. Fertility and Sterility 106:673-680.

DOI: $10.1016 /$ j.fertnstert.2016.05.020.

Cho S, Mutlu L, Zhou Y, Taylor HS. 2016b. Aromatase inhibitor regulates let-7 expression and let-7f-induced cell migration in endometrial cells from women with endometriosis. Fertility and Sterility 106:673-680.

DOI: 10.1016/j.fertnstert.2016.05.020.

Choi YS, Park JH, Lee JH, Yoon J-K, Yun BH, Park JH, Seo SK, Sung H-J, Kim H-S, Cho S, Lee BS. 2018. Association Between Impairment of DNA Double Strand Break Repair and Decreased Ovarian Reserve in Patients With Endometriosis. Frontiers in Endocrinology 9. DOI: 10.3389/fendo.2018.00772.

Deret S, Voegelin J, Lelong-Rebel IHC, Rebel G. 2004. Effects of culture conditions on taurine uptake by various variants of human endometrial carcinoma cells in culture. Amino Acids 26:183-195. DOI: $10.1007 / \mathrm{s} 00726-003-0035-7$.

Peer] reviewing PDF | (2020:04:47385:1:2:NEW 12 Aug 2020) 
421 Du Y, Zhang Z, Xiong W, Li N, Liu H, He H, Li Q, Liu Y, Zhang L. 2018. Estradiol promotes EMT in

422

423

424

425

426

427

428

429

430

431

432

433

434

435

436

437

438

439

440

441 endometriosis via MALAT1/miR200s sponge function. Reproduction (Cambridge, England). DOI: 10.1530/REP-18-0424.

Ehrlich M. 2009. DNA hypomethylation in cancer cells. Epigenomics 1:239-259. DOI: 10.2217/epi.09.33.

Filippi I, Carrarelli P, Luisi S, Batteux F, Chapron C, Naldini A, Petraglia F. 2016. Different Expression of Hypoxic and Angiogenic Factors in Human Endometriotic Lesions. Reproductive Sciences 23:492-497. DOI: $10.1177 / 1933719115607978$.

Fu H-L, Ma Y, Lu L-G, Hou P, Li B-J, Jin W-L, Cui D-X. 2014. TET1 exerts its tumor suppressor function by interacting with p53-EZH2 pathway in gastric cancer. Journal of Biomedical Nanotechnology 10:12171230. DOI: $10.1166 /$ jbn.2014.1861.

Giudice LC. 2010. Endometriosis. New England Journal of Medicine 362:2389-2398. DOI: 10.1056/NEJMcp1000274.

Guay S, Akoum A. 2007. Stable inhibition of interleukin 1 receptor type II in Ishikawa cells augments secretion of matrix metalloproteinases: possible role in endometriosis pathophysiology. Reproduction 134:525534.

Han X, Zhou Y, You Y, Lu J, Wang L, Hou H, Li J, Chen W, Zhao L, Li X. 2017. TET1 promotes cisplatinresistance via demethylating the vimentin promoter in ovarian cancer: TET1 in ovarian cancer. Cell Biology International 41:405-414. DOI: 10.1002/cbin.10734.

Hsiao K-Y. 2015. Pathological functions of hypoxia in endometriosis. Frontiers in Bioscience 7:352-366. DOI: $10.2741 / \mathrm{e} 736$

Hsu C-H, Peng K-L, Kang M-L, Chen Y-R, Yang Y-C, Tsai C-H, Chu C-S, Jeng Y-M, Chen Y-T, Lin F-M, 
442
Huang H-D, Lu Y-Y, Teng Y-C, Lin S-T, Lin R-K, Tang F-M, Lee S-B, Hsu HM, Yu J-C, Hsiao P-W, Juan L-J. 2012. TET1 suppresses cancer invasion by activating the tissue inhibitors of metalloproteinases. Cell Reports 2:568-579. DOI: 10.1016/j.celrep.2012.08.030.

Hu X-Q, Dasgupta C, Xiao J, Yang S, Zhang L. 2018. Long-term high altitude hypoxia during gestation suppresses large conductance $\mathrm{Ca} 2+$-activated $\mathrm{K}+$ channel function in uterine arteries: a causal role for microRNA-210. The Journal of Physiology 596:5891-5906. DOI: 10.1113/JP276058.

Huang Y, Wang G, Liang Z, Yang Y, Cui L, Liu C-Y. 2016. Loss of nuclear localization of TET2 in colorectal cancer. Clinical Epigenetics 8:9. DOI: 10.1186/s13148-016-0176-7.

Jain T, Nikolopoulou EA, Xu Q, Qu A. 2018. Hypoxia inducible factor as a therapeutic target for atherosclerosis. Pharmacology \& Therapeutics 183:22-33. DOI: 10.1016/j.pharmthera.2017.09.003.

Javaid S, Zhang J, Anderssen E, Black JC, Wittner BS, Tajima K, Ting DT, Smolen GA, Zubrowski M, Desai R, Maheswaran S, Ramaswamy S, Whetstine JR, Haber DA. 2013. Dynamic Chromatin Modification Sustains Epithelial-Mesenchymal Transition following Inducible Expression of Snail-1. Cell reports 5:1679-1689. DOI: 10.1016/j.celrep.2013.11.034.

Jeschke J, Collignon E, Fuks F. 2016. Portraits of TET-mediated DNA hydroxymethylation in cancer. Current Opinion in Genetics \& Development 36:16-26. DOI: 10.1016/j.gde.2016.01.004.

Jones PA. 2012. Functions of DNA methylation: islands, start sites, gene bodies and beyond. Nature Reviews. Genetics 13:484-492. DOI: 10.1038/nrg3230.

Juanqing L, Hailan Y, Xiangwei F, Tao Z, Junyan M, Jianhong Z, Jun L, Jianhua Y. 2019. Relationship between the methylation levels of Twist gene and pathogenesis of endometriosis. Cellular and Molecular Biology (Noisy-Le-Grand, France) 65:94-100.

Peer) reviewing PDF | (2020:04:47385:1:2:NEW 12 Aug 2020) 
463 Kaas GA, Zhong C, Eason DE, Ross DL, Vachhani RV, Ming G-L, King JR, Song H, Sweatt JD. 2013. TET1

464

465

466

467

468

469

470

471

472

473

474

475

476

477

478

479

480

481

482

483 controls CNS 5-methylcytosine hydroxylation, active DNA demethylation, gene transcription, and memory formation. Neuron 79:1086-1093. DOI: 10.1016/j.neuron.2013.08.032.

Koukoura O, Sifakis S, Spandidos DA. 2016. DNA methylation in endometriosis. Molecular medicine reports $13: 2939-2948$.

Lamouille S, Xu J, Derynck R. 2014. Molecular mechanisms of epithelial-mesenchymal transition. Nature reviews Molecular cell biology 15:178.

Lee M-Y, Kim SH, Oh YS, Heo S-H, Kim K-H, Chae HD, Kim C-H, Kang BM. 2018. Role of interleukin-32 in the pathogenesis of endometriosis: in vitro, human and transgenic mouse data. Human Reproduction 33:807-816. DOI: 10.1093/humrep/dey055.

Li Y, An D, Guan Y, Kang S. 2017. Aberrant Methylation of the E-Cadherin Gene Promoter Region in Endometrium and Ovarian Endometriotic Cysts of Patients with Ovarian Endometriosis. Gynecologic and Obstetric Investigation 82:78-85. DOI: 10.1159/000445293.

Lim YW, Sanz LA, Xu X, Hartono SR, Chédin F. 2015. Genome-wide DNA hypomethylation and RNA:DNA hybrid accumulation in Aicardi-Goutières syndrome. eLife 4. DOI: 10.7554/eLife.08007.

Lin G, Sun W, Yang Z, Guo J, Liu H, Liang J. 2017. Hypoxia induces the expression of TET enzymes in HepG2 cells. Oncology letters 14:6457-6462.

Logan PC, Yango P, Tran ND. 2018. Endometrial Stromal and Epithelial Cells Exhibit Unique Aberrant Molecular Defects in Patients With Endometriosis. Reproductive Sciences (Thousand Oaks, Calif.) 25:140-159. DOI: 10.1177/1933719117704905.

Lorsbach RB, Moore J, Mathew S, Raimondi SC, Mukatira ST, Downing JR. 2003. TET1, a member of a novel

Peer) reviewing PDF | (2020:04:47385:1:2:NEW 12 Aug 2020) 
484

485

486

487

488

489

490

491

492

493

494

495

496

497

498

499

500

501

502

503

504

protein family, is fused to MLL in acute myeloid leukemia containing the $\mathrm{t}(10 ; 11)(\mathrm{q} 22 ; \mathrm{q} 23)$. Leukemia $17: 637$.

Lv Q-Y, Xie B-Y, Yang B-Y, Ning C-C, Shan W-W, Gu C, Luo X-Z, Chen X-J, Zhang Z-B, Feng Y-J. 2017. Increased TET1 Expression in Inflammatory Microenvironment of Hyperinsulinemia Enhances the Response of Endometrial Cancer to Estrogen by Epigenetic Modulation of GPER. Journal of Cancer 8:894-902. DOI: 10.7150/jca.17064.

Mahmood TA, Templeton A. 1991. Prevalence and genesis of endometriosis. Human Reproduction 6:544-549. DOI: 10.1093/oxfordjournals.humrep.a137377.

Mariani CJ, Vasanthakumar A, Madzo J, Yesilkanal A, Bhagat T, Yu Y, Bhattacharyya S, Wenger RH, Cohn SL, Nanduri J. 2014. TET1-mediated hydroxymethylation facilitates hypoxic gene induction in neuroblastoma. Cell reports 7:1343-1352.

Matson BC, Quinn KE, Lessey BA, Young SL, Caron KM. 2018. Elevated levels of adrenomedullin in eutopic endometrium and plasma from women with endometriosis. Fertility and Sterility 109:1072-1078. DOI: 10.1016/j.fertnstert.2018.02.004.

Matsuzaki S, Darcha C. 2012. Epithelial to mesenchymal transition-like and mesenchymal to epithelial transition-like processes might be involved in the pathogenesis of pelvic endometriosis $\dagger$. Human Reproduction 27:712-721. DOI: 10.1093/humrep/der442.

Maybin JA, Critchley HOD. 2015. Menstrual physiology: implications for endometrial pathology and beyond. Human Reproduction Update 21:748-761. DOI: 10.1093/humupd/dmv038.

Mi Y, Gao X, Dai J, Ma Y, Xu L, Jin W. 2015. A Novel Function of TET2 in CNS: Sustaining Neuronal Survival. International Journal of Molecular Sciences 16:21846-21857. DOI: 10.3390/ijms160921846. 
505

506

507

508

509

510

511

512

513

514

515

516

517

518

519

520

521

522

523

524

525

Nisolle M, Donnez J. 1997. Peritoneal endometriosis, ovarian endometriosis, and adenomyotic nodules of the rectovaginal septum are three different entities. Fertility and Sterility 68:585-596. DOI: 10.1016/s00150282(97)00191-x.

Noë M, Ayhan A, Wang T-L, Shih I-M. 2018. Independent development of endometrial epithelium and stroma within the same endometriosis. The Journal of Pathology 245:265-269. DOI: 10.1002/path.5082.

Roca FJ, Loomans HA, Wittman AT, Creighton CJ, Hawkins SM. 2016. Ten-eleven translocation genes are downregulated in endometriosis. Current molecular medicine 16:288-298.

Shahrzad S, Bertrand K, Minhas K, Coomber B. 2007. Induction of DNA hypomethylation by tumor hypoxia. Epigenetics 2:119-125.

Song C-X, He C. 2012. Balance of DNA methylation and demethylation in cancer development. Genome Biology 13:173. DOI: 10.1186/gb-2012-13-10-173.

Song J, Wang W, Wang Y, Qin Y, Wang Y, Zhou J, Wang X, Zhang Y, Wang Q. 2019. Epithelial-mesenchymal transition markers screened in a cell-based model and validated in lung adenocarcinoma. BMC Cancer 19:680. DOI: 10.1186/s12885-019-5885-9.

Tsai Y-P, Chen H-F, Chen S-Y, Cheng W-C, Wang H-W, Shen Z-J, Song C, Teng S-C, He C, Wu K-J. 2014. TET1 regulates hypoxia-induced epithelial-mesenchymal transition by acting as a co-activator. Genome biology 15:513. DOI: 10.1186/s13059-014-0513-0.

Tsai Y-P, Wu K-J. 2014. Epigenetic regulation of hypoxia-responsive gene expression: Focusing on chromatin and DNA modifications. International Journal of Cancer 134:249-256. DOI: 10.1002/ijc.28190.

Wu M-H, Chen K-F, Lin S-C, Lgu C-W, Tsai S-J. 2007. Aberrant expression of leptin in human endometriotic stromal cells is induced by elevated levels of hypoxia inducible factor-1 $\alpha$. The American journal of 
pathology 170:590-598.

527

528

529

530

531

532

533

534

535

536

537

538

539

540

541

542

Wu M-H, Hsiao K-Y, Tsai S-J. 2019. Hypoxia: The force of endometriosis. Journal of Obstetrics and Gynaecology Research 45:532-541. DOI: 10.1111/jog.13900.

Wu C-Y, Tsai Y-P, Wu M-Z, Teng S-C, Wu K-J. 2012. Epigenetic reprogramming and post-transcriptional regulation during the epithelial-mesenchymal transition. Trends in Genetics 28:454-463. DOI: 10.1016/j.tig.2012.05.005.

Wu X, Zhang Y. 2017. TET-mediated active DNA demethylation: mechanism, function and beyond. Nature Reviews Genetics 18:517.

Xiong Y, Liu Y, Xiong W, Zhang L, Liu H, Du Y, Li N. 2016. Hypoxia-inducible factor $1 \alpha$-induced epithelialmesenchymal transition of endometrial epithelial cells may contribute to the development of endometriosis. Human Reproduction 31:1327-1338. DOI: 10.1093/humrep/dew081.

Yang R, Qu C, Zhou Y, Konkel JE, Shi S, Liu Y, Chen C, Liu S, Liu D, Chen Y. 2015. Hydrogen sulfide promotes Tet1-and Tet2-mediated Foxp3 demethylation to drive regulatory $\mathrm{T}$ cell differentiation and maintain immune homeostasis. Immunity 43:251-263.

Zhou J, Schmid T, Schnitzer S, Brüne B. 2006. Tumor hypoxia and cancer progression. Cancer letters 237:1021. 
Figure 1

An infographic of this work

Hypoxia may increase TET1 expression, mediated by HIF-2 $\alpha$, thus potentially inducing the EMT of endometrial epithelial cells and contributing to the development of endometriosis
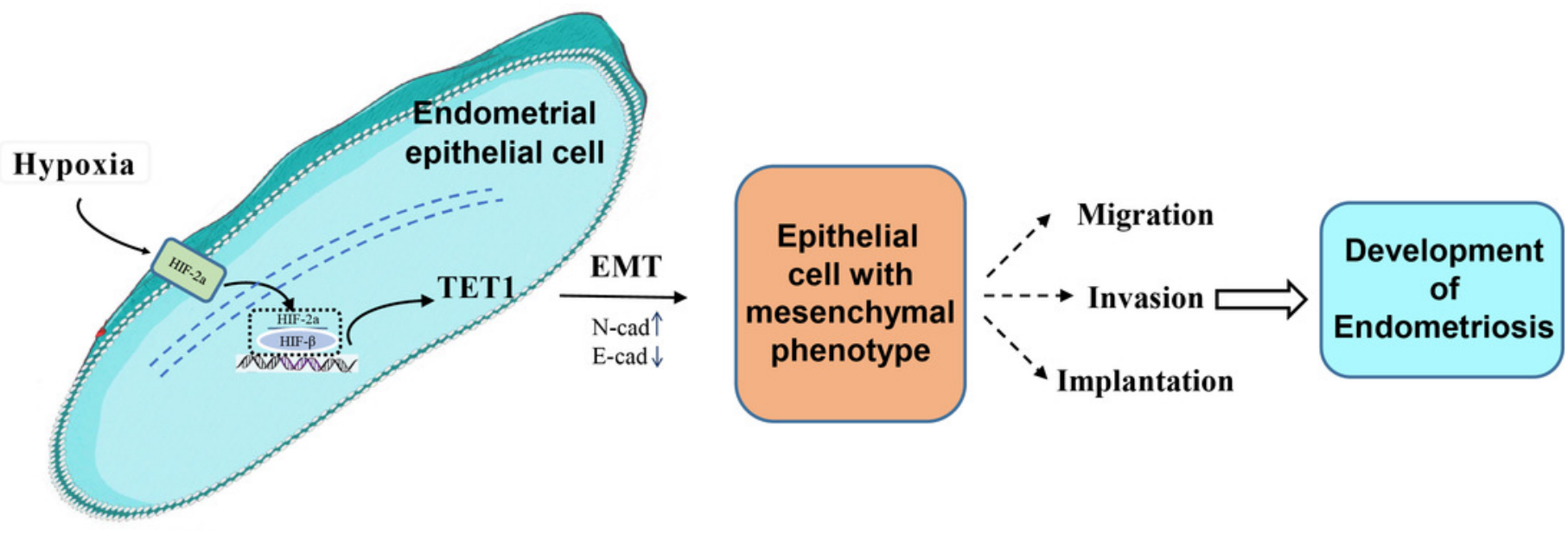


\section{Figure 2}

Accompanied with the TET1 upregulation, EMT might occur in endometrial epithelial cells of ovarian endometriosis.

Fig. 1 Accompanied with TET1 upregulation, EMT might occur in endometrial epithelial cells of ovarian endometriosis. (A-R) Immunohistochemistry for TET1 and EMT markers (Ecadherin and $\mathrm{N}$-cadherin) in epithelial gland cells of the normal endometria (Normal), eutopic endometria (Eutopic) and ovarian endometrioma (Ectopic). Left: Magnification $\times 100$, Right: Magnification $\times 200$. (S-U) The proportions of cells positively stained for TET1, E-cadherin or $\mathrm{N}$-cadherin in Normal, Eutopic and Ectopic groups $(* p<0.05$ versus normal endometria; $\# p<$ 0.05 versus eutopic endometria). (V) Expression of global 5-hmC in the total genomic DNA ( ${ }^{*} p<0.05$ versus normal endometria; $\# p<0.05$ versus eutopic endometria). (W-EE) Representative double immunofluorescence images for TET1 and E-cadherin in Normal (WCC), Eutopic (Z-BB) and Ectopic (CC-EE) groups. (FF-NN) Representative double immunofluorescence images for TET1 and N-cadherin in Normal (FF-HH), Eutopic (II-KK) and Ectopic (LL-NN) groups. (OO-PP) The correlation between TET1 and EMT markers expression in the endometrial epithelium is confirmed by quantitative analysis of the double staining immunofluorescence. Magnification $\times 200$ (Left: red luminescence represents TET1. Yellow luminescence represents E-cadherin. Right: red luminescence represents TET1. Green luminescence represents N-cadherin). Normal: normal endometria, Eutopic: eutopic endometria, Ectopic: ovarian endometriomas. 
TET1

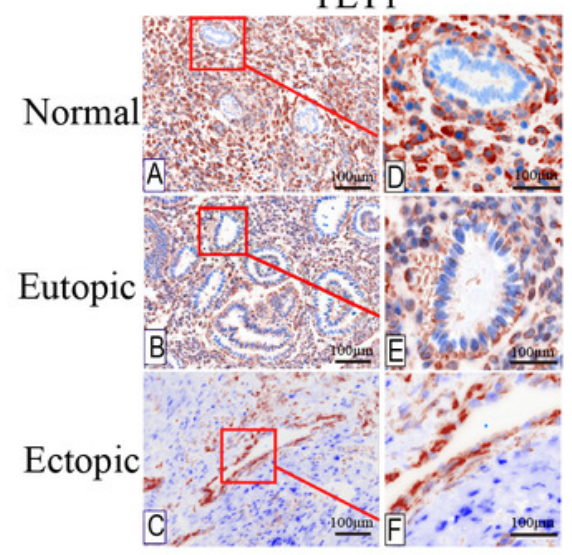

E-cadherin

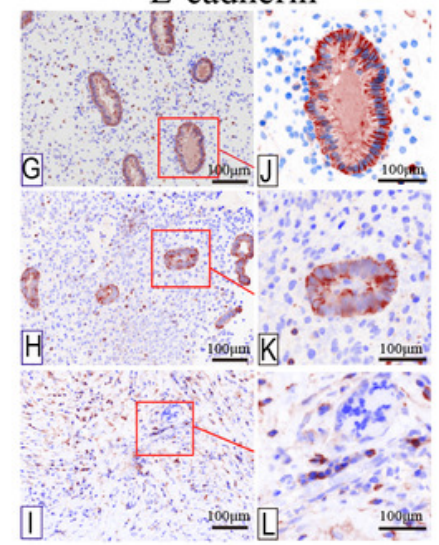

$\mathrm{N}$-cadherin

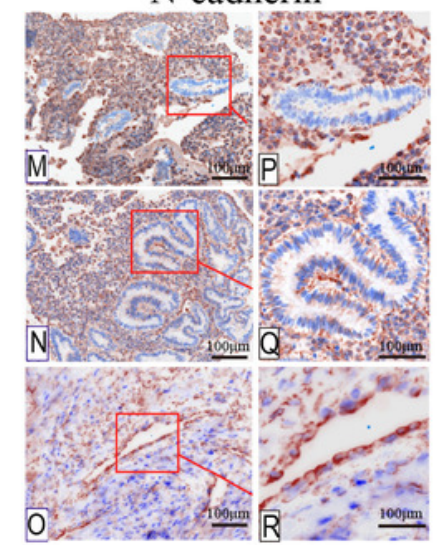

S

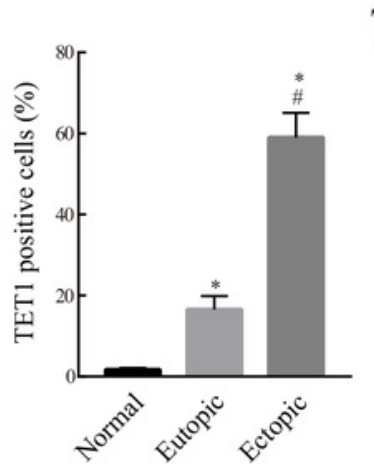

$\mathrm{T}$

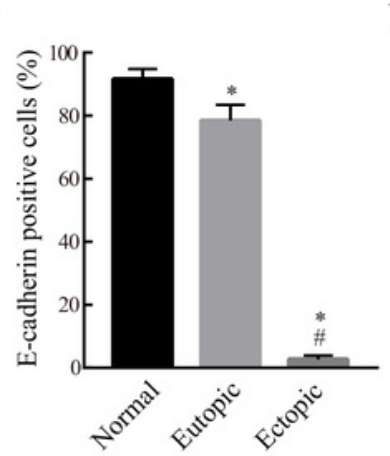

$\mathrm{U}$

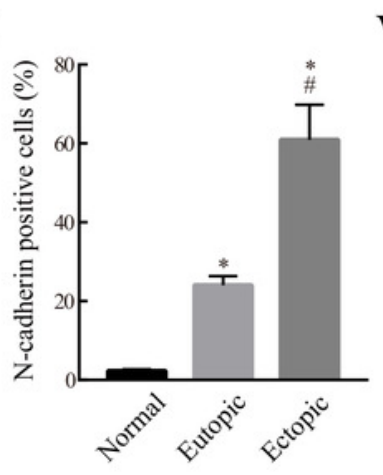

V

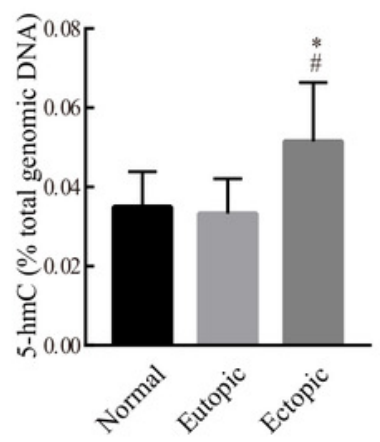

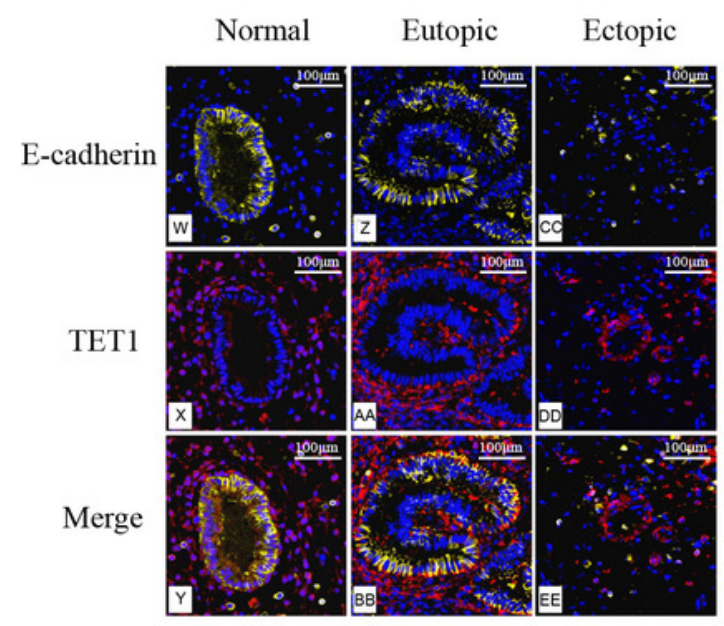
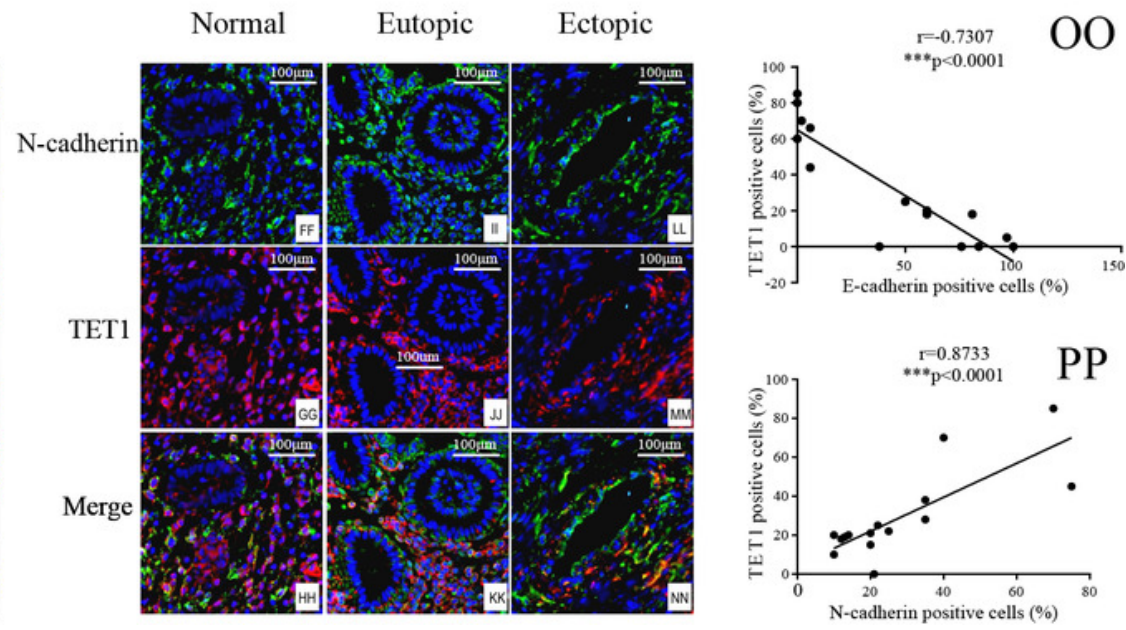
Figure 3

Hypoxia alters the expression of HIFs, TET1 and EMT markers.

(A-B)Western blotting analysis showed the expression of HIF-1 $\alpha$, HIF-2 $\alpha$, TET1 and EMT markers (E-Cad: E-cadherin, N-Cad: N-cadherin and Vim: Vimentin) in Ishikawa(ISK) cell cultured under hypoxia at different time point $(0 h, 4 h, 8 \mathrm{~h}$ and $24 \mathrm{~h})\left({ }^{*} p<0.05\right.$ versus normoxia; $\# p<0.05$ versus hypoxia for $4 h ; \& p<0.05$ versus hypoxia for $8 h$ ).

A

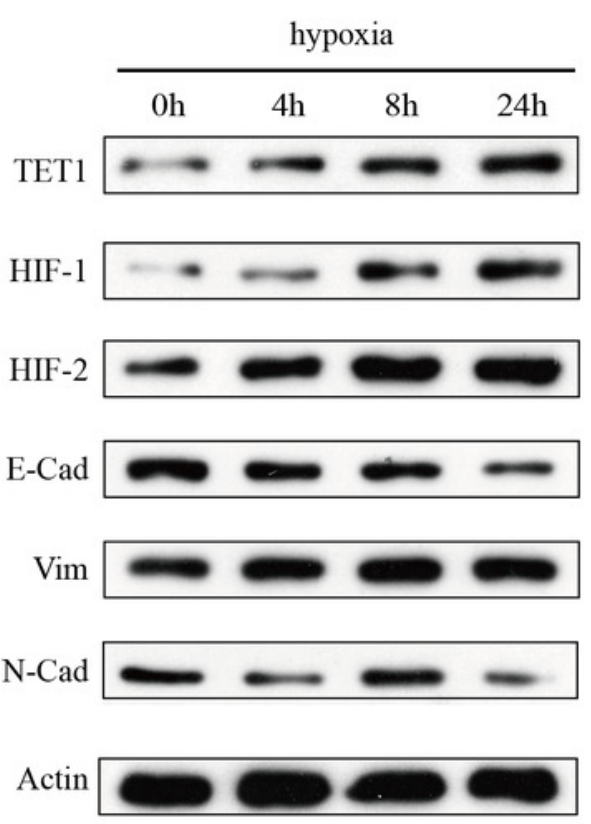

B

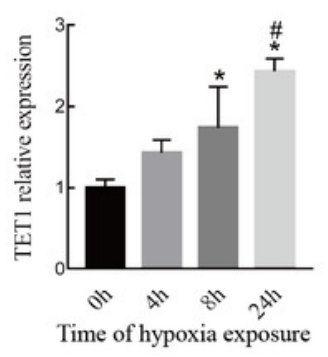

E

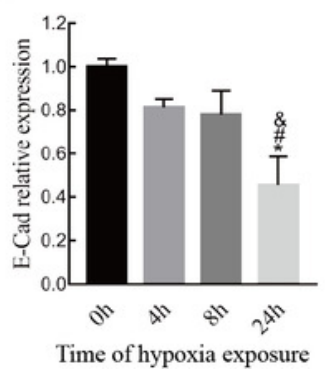

C

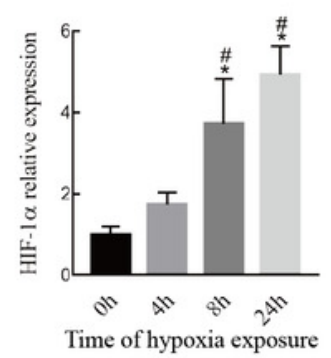

F

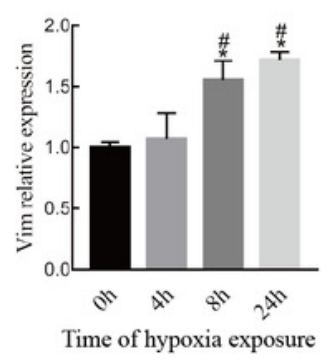

D

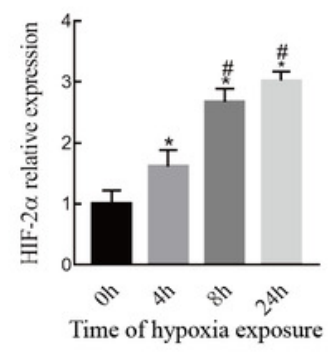

G

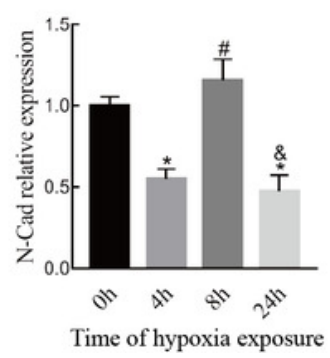


Figure 4

TET1 overexpression may promote EMT and knockdown of TET1 mitigates hypoxiainduced EMT.

(A, B) Western blotting analysis showed the expression of vimentin (Vim) and E-cadherin (ECad) in cells transfected with TET1 expression plasmid (TET1) or empty vectors (NC) under normoxia conditions, and cells transfected with TET small interfering RNA (si-TET1) or negative control (si-NC) under hypoxia conditions. $\left({ }^{*} p<0.05\right.$ versus normoxia $+N C ; \# p<0.05$ versus normoxia+TET1; $\& p<0.05$ versus hypoxia+si-NC).

A
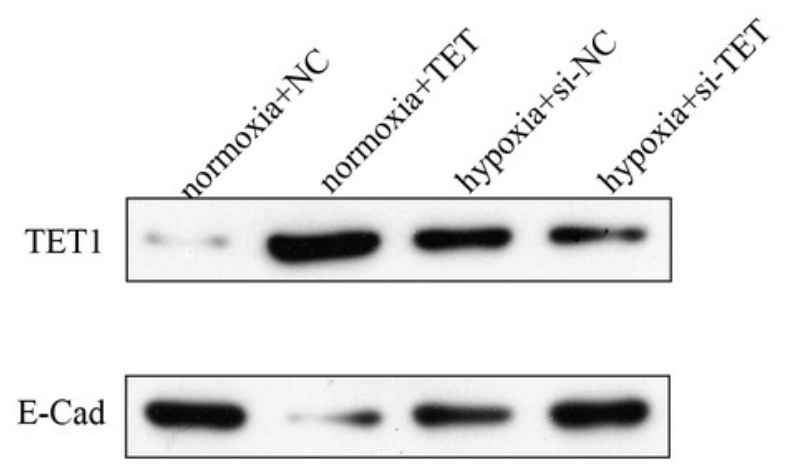

Vim

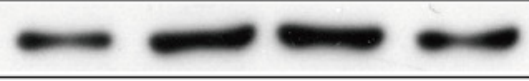

Actin

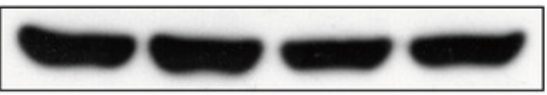

$\mathrm{B}$

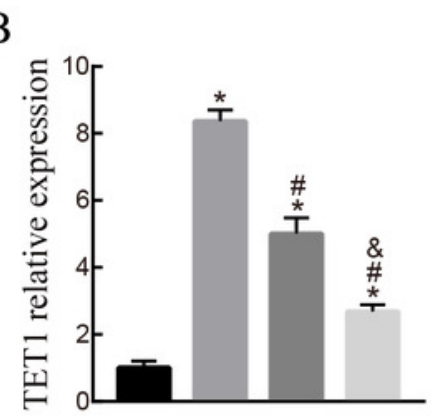

$\mathrm{C}$

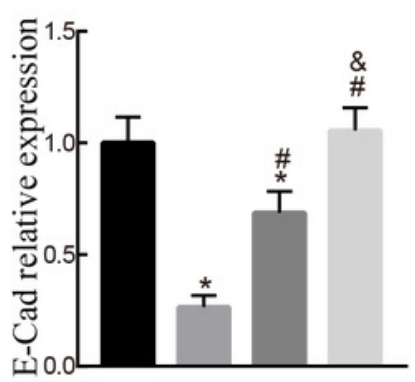

D

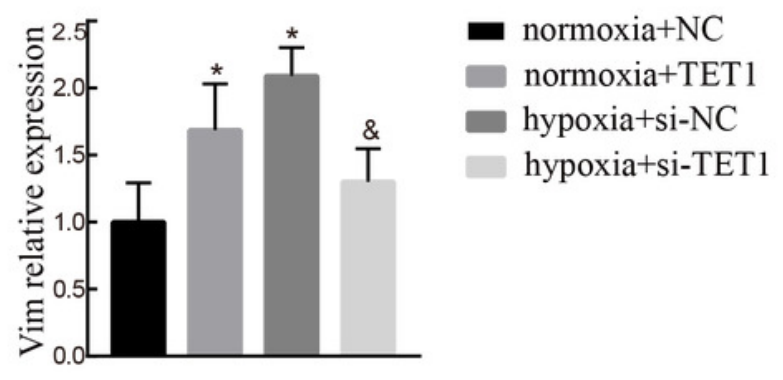




\section{Figure 5}

Knockdown of HIF-2 $\alpha$ inhibits hypoxia-induced EMT and TET1 expression.

(A, C) Western blotting showed the expression of TET1, HIF-2a, vimentin (Vim) and Ecadherin (E-Cad) in cells under normoxia conditions and cells transfected with HIF-2a small interfering RNA (si-HIF-2a) or negative control (si-NC) under hypoxia conditions $\left({ }^{*} p<0.05\right.$ versus normoxia or normoxia+si-NC; $\# p<0.05$ versus hypoxia or hypoxia+si-NC). (B) Coimmunoprecipitation of TET1, HIF-2 $\alpha$ or HIF-1 $\alpha$. Hypoxia treated ISK cells were lysed and immunopreciated by TET1, HIF-2 $\alpha$, HIF-1 $\alpha$ and normal rabbit/mouse IgG antibodies, respectively. Cell lysates (input) and immunoprecipitated proteins (IP) were analyzed by Western blotting. 
A

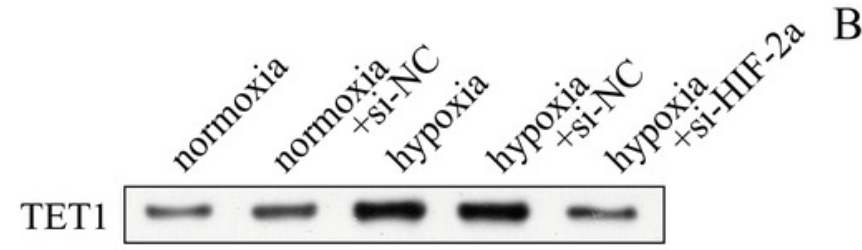

HIF-2a

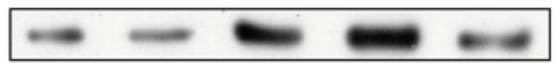

E-Cad
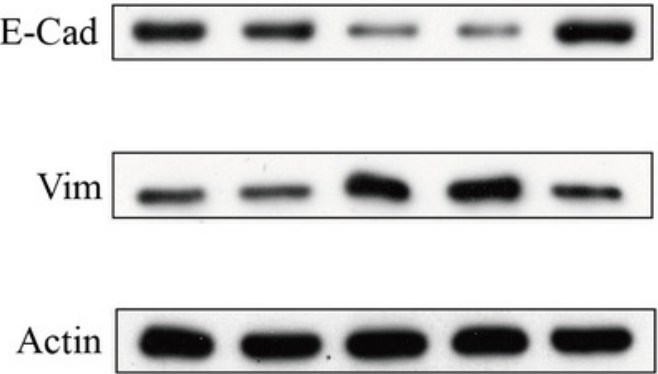

B

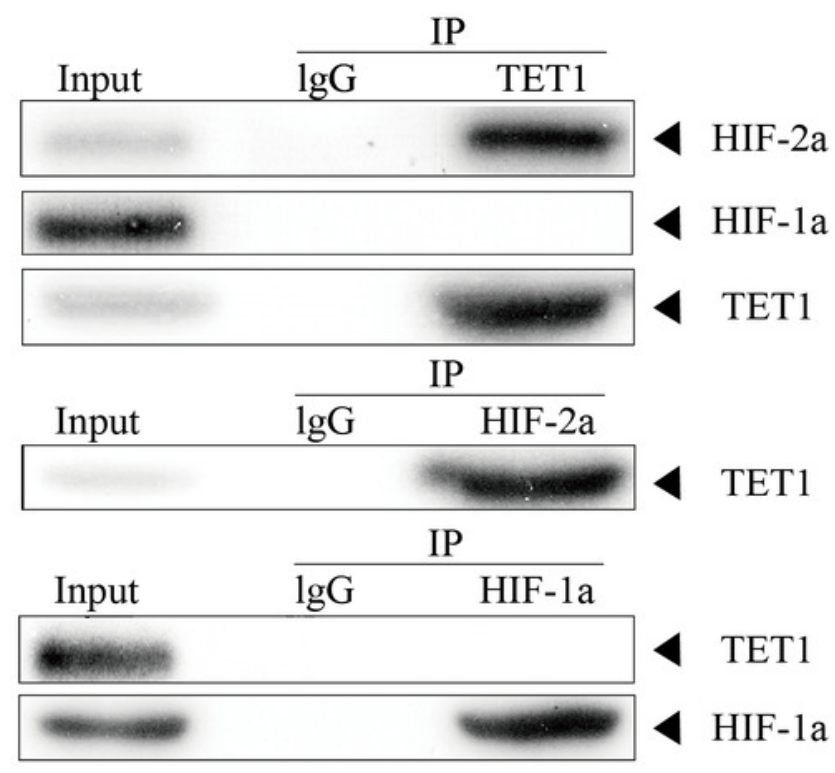

E

F normoxia

hypoxia

hypoxia+si-NC
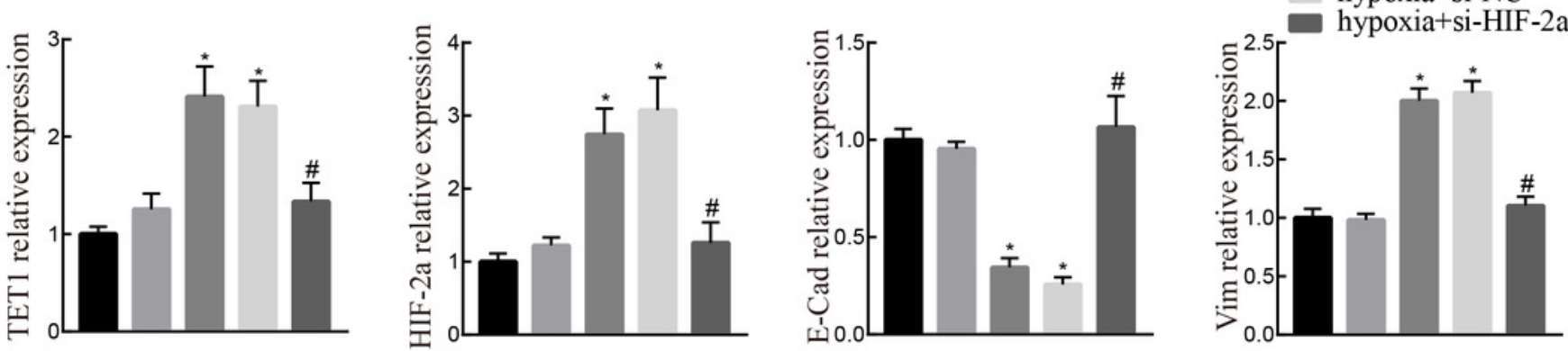
Table $\mathbf{1}$ (on next page)

Clinical characteristics of patients 
Table 1 Clinical characteristics of patients.

\begin{tabular}{cccc}
\hline & \multicolumn{3}{c}{ Immunohistochemistry samples } \\
\cline { 2 - 4 } & Normal endometria & Eutopic endometria & Ectopic endometria \\
\hline case number & 20 & 15 & 15 \\
$*$ age & $35(27-41)$ & $29(27-38)$ & $29(27-38)$ \\
menstrual cycle phase & Early proliferation & Early proliferation & Early proliferation \\
\#rASRM stage & & & \\
III & - & 9 & 6 \\
IV & - & 6 & 6 \\
\hline
\end{tabular}

Eutopic endometria were paired with ectopic endometria.

*Medßan (interquartile range).

\# rASRM (Revised American Society for Reproductive Medicine classification, 1997).

5

6

7 


\section{Table 2 (on next page)}

Expression profiles of TET1 and EMT markers were detected by immunohistochemistry in the epithelium of normal endometria, eutopic endometria, and ectopic endometria. 
Table 2 Expression profiles of TET1 and EMT markers were detected by immunohistochemistry in the epithelia of normal endometria, eutopic endometria, and ectopic endometria.

\begin{tabular}{|c|c|c|c|c|c|c|}
\hline \multirow[b]{2}{*}{ Marker } & \multicolumn{3}{|c|}{ Positive epithelial cells for marker, $\%$} & \multirow[b]{2}{*}{$\begin{array}{c}p \text {-value } \\
(1 \text { versus } 2)\end{array}$} & \multirow[b]{2}{*}{$\begin{array}{c}\text { p-value } \\
\text { (2 versus } 3)\end{array}$} & \multirow[b]{2}{*}{$\begin{array}{c}p \text {-value } \\
(1 \text { versus } 3)\end{array}$} \\
\hline & $\begin{array}{c}\text { Normal } \\
\text { endometria, \% } \\
\text { (group1,n=15) }\end{array}$ & $\begin{array}{c}\text { Eutopic } \\
\text { endometria, \% } \\
\text { (group2,n=15) }\end{array}$ & $\begin{array}{c}\text { Ectopic } \\
\text { endometria, \% } \\
\text { (group3,n=15) }\end{array}$ & & & \\
\hline E-cadherin & $91.66 \pm 3.196$ & $77.75 \pm 4.024$ & $2.668 \pm 1.174$ & $<0.05^{*}$ & $<0.01^{* *}$ & $<0.01^{* *}$ \\
\hline $\mathrm{N}$-cadherin & $2.235 \pm 0.339$ & $24.11 \pm 2.237$ & $60.98 \pm 8.858$ & $<0.01^{* *}$ & $<0.01^{* *}$ & $<0.01^{* *}$ \\
\hline TET1 & $1.668 \pm 0.438$ & $16.57 \pm 3.308$ & $59.03 \pm 6.042$ & $<0.01^{* *}$ & $<0.01^{* *}$ & $<0.01^{* *}$ \\
\hline
\end{tabular}

$* \mathrm{P}<0 . \mathrm{B}) 5, * * \mathrm{P}<0.01$

All data are expressed as mean $+\mathrm{SD}$.

5

6

7

8

9 